\title{
The Wise Gamma-ray Strip Parameterization: The Nature of the Gamma-ray Active Galactic Nuclei of Uncertain Type
}

\section{Citation}

Massaro, F., R. D’Abrusco, G. Tosti, M. Ajello, D. Gasparrini, J. E. Grindlay, and Howard A. Smith. 2012. "THE WISE GAMMA-RAY STRIP PARAMETERIZATION: THE NATURE OF THE GAMMARAY ACTIVE GALACTIC NUCLEI OF UNCERTAIN TYPE." The Astrophysical Journal 750 (2): 138. https://doi.org/10.1088/0004-637x/750/2/138.

\section{Permanent link}

http://nrs.harvard.edu/urn-3:HUL.InstRepos:41399799

\section{Terms of Use}

This article was downloaded from Harvard University's DASH repository, and is made available under the terms and conditions applicable to Other Posted Material, as set forth at http:// nrs.harvard.edu/urn-3:HUL.InstRepos:dash.current.terms-of-use\#LAA

\section{Share Your Story}

The Harvard community has made this article openly available.

Please share how this access benefits you. Submit a story.

\section{Accessibility}




\title{
THE WISE GAMMA-RAY STRIP PARAMETERIZATION: THE NATURE OF THE GAMMA-RAY ACTIVE GALACTIC NUCLEI OF UNCERTAIN TYPE
}

\author{
F. Massaro ${ }^{1}$, R. D’Abrusco ${ }^{2}$, G. Tosti ${ }^{3,4}$, M. Ajello ${ }^{1}$, D. Gasparrini ${ }^{5}$, J. E. Grindlay ${ }^{2}$, And Howard A. Smith ${ }^{2}$ \\ ${ }^{1}$ SLAC National Laboratory and Kavli Institute for Particle Astrophysics and Cosmology, 2575 Sand Hill Road, Menlo Park, CA 94025, USA \\ ${ }^{2}$ Harvard-Smithsonian Astrophysical Observatory, 60 Garden Street, Cambridge, MA 02138, USA \\ ${ }^{3}$ Dipartimento di Fisica, Università degli Studi di Perugia, 06123 Perugia, Italy \\ ${ }^{4}$ Istituto Nazionale di Fisica Nucleare, Sezione di Perugia, 06123 Perugia, Italy \\ ${ }^{5}$ ASI Science Data Center, ESRIN, I-00044 Frascati, Italy \\ Received 2012 January 20; accepted 2012 February 23; published 2012 April 25
}

\begin{abstract}
Despite the large number of discoveries made recently by Fermi, the origins of the so-called unidentified $\gamma$-ray sources remain unknown. The large number of these sources suggests that there could be a population among them that significantly contributes to the isotropic gamma-ray background and it is therefore crucial to understand their nature. The first step toward a complete comprehension of the unidentified $\gamma$-ray source population is to identify those that can be associated with blazars, the most numerous class of extragalactic sources in the $\gamma$-ray sky. Recently, we discovered that blazars can be recognized and separated from other extragalactic sources using the infrared (IR) WISE satellite colors. The blazar population delineates a remarkable and distinctive region of the IR color-color space, the WISE blazar strip. In particular, the subregion delineated by the $\gamma$-ray emitting blazars is even narrower and we named it the WISE Gamma-ray Strip (WGS). In this paper, we parameterize the WGS on the basis of a single parameter $s$ that we then use to determine if $\gamma$-ray active galactic nuclei of the uncertain type (AGUs) detected by Fermi are consistent with the WGS and can be considered blazar candidates. We find that 54 AGUs out of a set of 60 analyzed have IR colors consistent with the WGS; only 6 AGUs are outliers. This result implies that a very high percentage (i.e., in this sample about 90\%) of the AGUs detected by Fermi are indeed blazar candidates.
\end{abstract}

Key words: BL Lacertae objects: general - galaxies: active - gamma rays: general - radiation mechanisms: non-thermal

Online-only material: color figures

\section{INTRODUCTION}

With the recent advent of the Fermi mission, the $\gamma$-ray astronomy is living a new golden age with several striking discoveries already performed during the first three years.

According to the second Fermi $\gamma$-ray LAT catalog (2FGL; Nolan et al. 2012), Fermi detected 1873 sources, 576 of which are still unidentified even if the localization of the $\gamma$-ray sources has significantly improved with respect to the past $\gamma$-ray missions. For this reason, despite the large number of new discoveries already achieved, the nature of the unidentified $\gamma$-ray sources is still an open question. This unsolved issue is extremely relevant for the origin of the isotropic gamma-ray background, since, given the large number of unidentified $\gamma$-ray sources, new classes of unknown extragalactic $\gamma$-ray sources that can significantly contribute to the isotropic gamma-ray background could be hidden.

On the other hand, the most detected $\gamma$-ray sources in the $\mathrm{MeV}-\mathrm{GeV}$ energy range belong to the rarest class of active galactic nuclei, the blazars. They are an intriguing class of active galactic nuclei, characterized by non-thermal radiation emitted over the entire electromagnetic spectrum, and interpreted as arising from a relativistic jet closely aligned to the line of sight (see, e.g., Blandford \& Rees 1978). Blazars come in two flavors: the BL Lac objects and the flat spectrum radio quasars, where the common discriminating criterion between the two classes is the equivalent width of the optical emission lines, traditionally weaker than $5 \AA$ in the former rather than in the latter (Stickel et al. 1991; Stoke et al. 1991). In the following, we refer to the BL Lacs as BZBs and the flat spectrum radio quasars as BZQs, according to the ROMA-BZCAT nomenclature (Massaro et al. 2009, 2010, 2011).

Recently, using the preliminary data release of the WISE infrared (IR) survey (Wright et al. 2010), we discovered that IR color-color diagrams allow us to distinguish between extragalactic sources dominated by non-thermal emission, like blazars, and other classes of galaxies and/or active galactic nuclei (Massaro et al. 2011, hereafter Paper I; see also Plotkin et al. 2011). In particular, the blazar population delineates a tight, distinct region of the IR color space, indicated as the WISE Blazar Strip (Paper I). The WISE Blazar Strip is a region in the 3D infrared color space delineated by the blazar population. This region is narrower when considering only the IR colors of the blazars that are detected in the $\gamma$-rays, indicated as the WISE gamma-ray strip (WGS; see D'Abrusco et al. 2012, hereafter Paper II). A 3D scatter plot of the WISE Blazar Strip and the subregion of the WGS are shown in the IR diagram of Figure 1, while the [3.4]-[4.6]-[12] $\mu \mathrm{m} 2 \mathrm{D}$ projection is reported in Figure 2.

One of the major difficulties of the association procedures for the Fermi $\gamma$-ray sources with active galactic nuclei is that, due to the lack of radio and X-ray information and to the large uncertainty on the $\gamma$-ray position, it is not always possible to recognize if there is a blazar candidate within the positional error region. Thus, the main aim of this paper is to build a parameterization of the WGS in order to verify whenever $\gamma$-ray sources have been associated with a counterpart that is a blazar candidate, being consistent with the WGS. In particular, we studied the WISE counterparts of the active galactic nuclei of uncertain type (AGUs), defined according to the $2 \mathrm{FGL}$ and the 


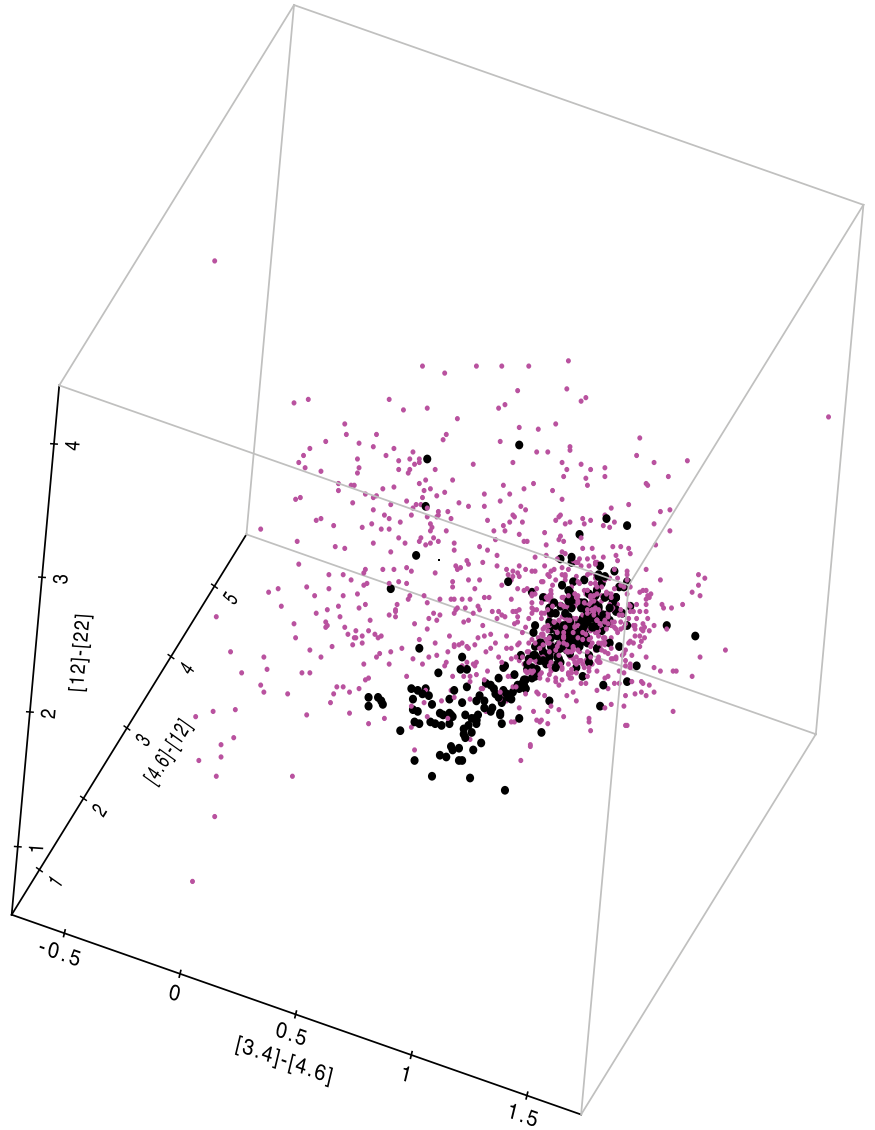

Figure 1. 3D representation of the WISE Blazar Strip (blazars are indicated in magenta) and the subregion of the WGS ( $\gamma$-ray emitting blazars are indicated in black) in the IR colors built with the magnitudes in the WISE bands at [3.4]-[4.6]-[12]-[22] $\mu \mathrm{m}$.

(A color version of this figure is available in the online journal.)

2LAC criteria (Ackermann et al. 2011), and their consistency with the WGS. The AGUs are defined as the radio and/or $\mathrm{X}$-ray counterparts of $\gamma$-ray sources associated with by the 2FGL likelihood ratio method, but without a good optical spectrum that enable their classification (Nolan et al. 2012). This paper is organized as follows. Section 2 describes the procedure adopted to parameterize the WGS. In Section 3, we discuss on the consistency of the WISE counterparts of the AGU sample with the WGS, while Section 4 describes the non-parametric analysis of the WGS based on the kernel density estimation (KDE). In Section 5, we investigated possible selection effects that could affect our WGS parameterization. Our conclusions are discussed in Section 6.

\section{THE WISE GAMMA-RAY STRIP PARAMETERIZATION}

\subsection{The Sample Selection}

We use the sample of $\gamma$-ray emitting blazars already selected in Paper II to parameterize the WGS.

This sample was selected from the 2FGL that contains 805 sources associated with a blazar: $435 \mathrm{BZBs}$ and $370 \mathrm{BZQs}$, respectively. However, only 659 (347 BZBs and 312 BZQs) of these are listed and classified according to the criteria used in the ROMA-BZCAT (e.g., Massaro et al. 2009). We excluded from our analysis all the blazars with a Fermi analysis flag, according to the 2FGL and the 2LAC (Nolan et al. 2012; Ackermann et al. 2011).

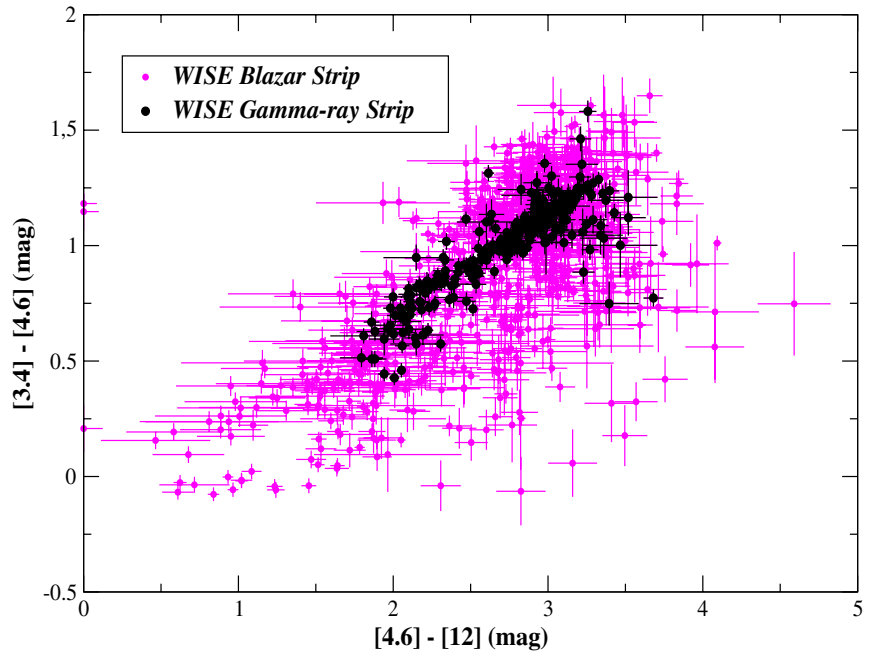

Figure 2. 2D projection of the WISE Blazar Strip (blazars are in magenta) and the subregion of the WGS ( $\gamma$-ray emitting blazars are in black) in the IR color diagram [3.4]-[4.6]-[12] $\mu \mathrm{m}$.

(A color version of this figure is available in the online journal.)

In particular, 329 (164 BZBs and 165 BZQs) blazars out of the original 659 lie in the portion of the sky reported in the WISE preliminary source catalog, but only 296 (143 BZBs and 153 BZQs) have a WISE counterpart within 2."4 radius (see Paper I). To be more conservative, we excluded from our analysis 12 blazars ( 8 BZBs and 4 BZQs) with respect to the 296 blazars in sample selected in Paper II, because they have a 95\% upper limit on the WISE magnitude at $22 \mu \mathrm{m}$. Then, we use this $2 \mathrm{FB}$ sample composed of the 284 blazars (135 BZBs and 149 BZQs) to build the WGS parameterization. We note that all the selected blazars also belong to the 2LAC sample (Ackermann et al. 2011).

According to the classification available in ROMA-BZCAT, the blazars of uncertain type have been excluded from our analysis, while the BL Lac candidates have been considered as BZBs. More details about the 2FB sample and the source selections are given in Paper II.

Finally, we emphasize that our selection is based only on $\gamma$-ray blazars that belong to the ROMA-BZCAT because this is the largest catalog of blazars available in the literature in which each source is spectroscopically classified at optical frequencies.

\subsection{The WISE Blazar Associations}

The IR color-color diagrams have been built using the archival WISE Preliminary Source Catalog, that covers $\sim 57 \%$ of the sky. ${ }^{6}$ The WISE mission mapped the sky at 3.4, 4.6, 12, and $22 \mu \mathrm{m}$ in 2010 with an angular resolution of $6.1,6.4,6.5$, and $12^{\prime \prime} .0$ in the four bands, achieving $5 \sigma$ point-source sensitivities of $0.08,0.11,1$, and $6 \mathrm{mJy}$, respectively, in unconfused regions on the ecliptic. All the WISE magnitudes are in the Vega system. In particular, the absolute (radial) differences between WISE source-peaks and "true" astrometric positions anywhere on the sky are no larger than $\sim 0^{\prime} .50,0.26,0$.'26, and $1^{\prime \prime} .4$ in the four WISE bands, respectively (Cutri et al. 2011). ${ }^{7}$

For our analysis, unless stated otherwise, we considered only WISE sources detected with a minimum signal-to-noise ratio of 7 in at least one band. The positional coincidences of blazars in the observed WISE sky have been searched within a circular

\footnotetext{
6 wise2.ipac.caltech.edu/docs/release/prelim/preview.html

7 wise2.ipac.caltech.edu/docs/release/prelim/expsup/sec2_3g.html
} 


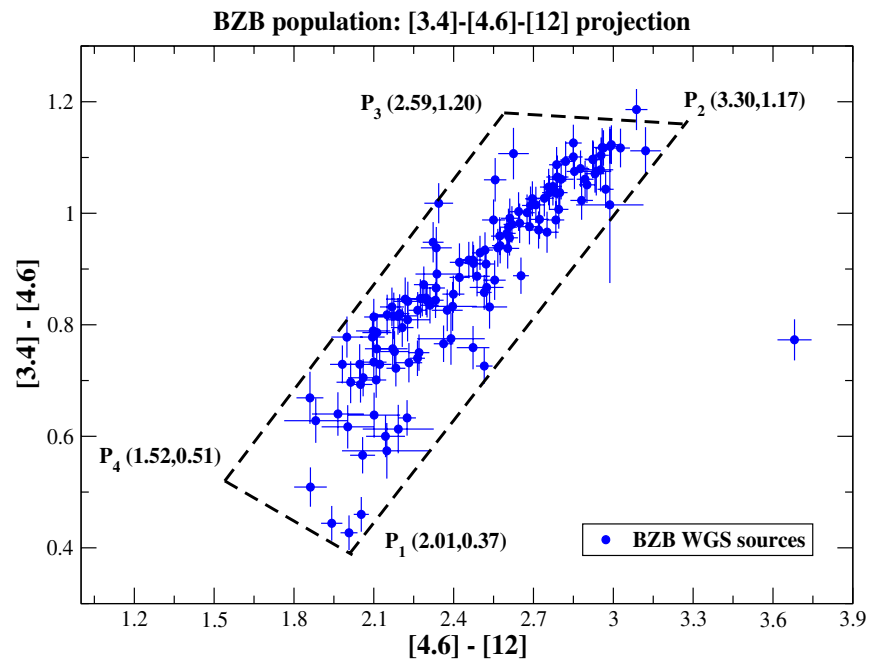

Figure 3. The [3.4]-[4.6] $-[12] \mu \mathrm{m} 2 \mathrm{D}$ projection of the WGS in the subregion of the BZB population is shown.

(A color version of this figure is available in the online journal.)

region of radius 2 .'4. This corresponds to the combination of the error of $1^{\prime \prime}$, assumed for the radio position reported in the ROMA-BZCAT (Massaro et al. 2009) and taking into account astrometric uncertainties in the WISE preliminary data, and the positional error of the fourth WISE band at $22 \mu \mathrm{m}$ (i.e., 1".4; see also Paper I). All the associations of the 2FB blazars with WISE sources are unique and no multiple matches have been found (see Papers I and II for more details). The chance probabilities of the WISE associations for the sources in the 2FGL and in the ROMA-BZCAT are reported in Paper II.

\subsection{The WISE Gamma-ray Strip Projections}

We built the parameterization of the WGS considering only the sources in the 2FB sample (see Section 2.1) and using the three different 2D projections of the WGS delineated in the [3.4]-[4.6]-[12] $\mu \mathrm{m},[4.6]-[12]-[22] \mu \mathrm{m}$, and [3.4]-[4.6]-[22] $\mu \mathrm{m}$ color-color planes. In each color-color 2D projection we determined the smaller irregular quadrilateral containing at least $95 \%$ of the blazars in the $2 \mathrm{FB}$ sample considering their position within error (see also Section 2.4 for more details). The irregular quadrilateral defining the WGS subregions have been drawn by hand. The KDE analysis has been used to verify, a posteriori, that the hand-drawn boundaries of the WGS are in agreement with the sharp decline in density of WGS sources, as evaluated by this non-parametric method (see Section 4 for more details). This WGS modeling has been developed separately for the BZBs and the BZQs, and for all their three 2D projections.

The [3.4]-[4.6]-[12] $\mu \mathrm{m},[4.6]-[12]-[22] \mu \mathrm{m}$, and [3.4][4.6]-[12]-[22] $\mu \mathrm{m} 2 \mathrm{D}$ projections of the WGS for the BZB and the BZQ populations are shown in Figures 3-6, respectively.

In the following, we also report the boundaries chosen for our WGS parameterization. For the BZB projections the extremal points of the WGS have the coordinates: $P_{1}=(2.01,0.37)$, $P_{2}=(3.30,1.17), P_{3}=(2.59,1.20), P_{4}=(1.52,0.51)$ in Figure $3 ; P_{1}=(2.20,1.65), P_{2}=(2.72,2.57), P_{3}=(2.29,3.30)$, $P_{4}=(1.20,1.96)$ in Figure 5 (upper panel); while $P_{1}=(2.05$, $0.33), P_{2}=(2.83,1.07), P_{3}=(2.28,1.21), P_{4}=(1.20,0.73) \mathrm{in}$ Figure 5 (lower panel).

On the other hand, for the BZQ projections the extremal points of the WGS have the coordinates: $P_{1}=(2.90,0.85), P_{2}=(3.81$,

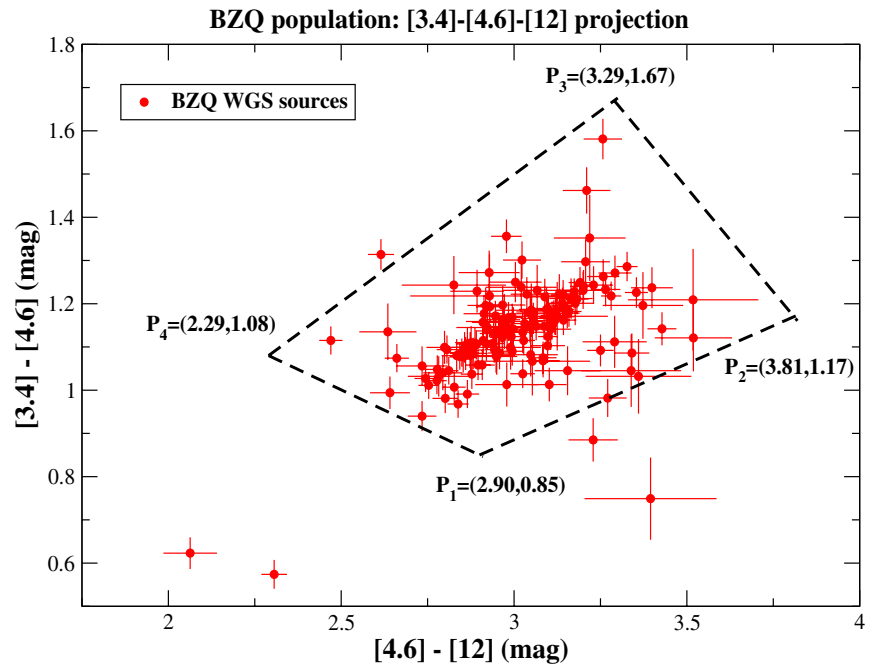

Figure 4. Same as Figure 3 but for the case of the BZQ population. (A color version of this figure is available in the online journal.)

1.17), $P_{3}=(3.29,1.67), P_{4}=(2.29,1.08)$ in Figure $4 ; P_{1}=$ $(2.25,2.22), P_{2}=(3.04,3.05), P_{3}=(2.67,3.70), P_{4}=(1.68$, 2.85 ) in Figure 6 (upper panel); while $P_{1}=(2.48,0.78), P_{2}=$ $(3.05,1.17), P_{3}=(2.55,1.50), P_{4}=(1.72,1.12)$ in Figure 6 (lower panel).

\subsection{The Strip Parameter $s$}

To illustrate the WGS parameterization, we consider the schematic case of the first projection: [3.4]-[4.6], [4.6]-[12], hereinafter $c_{1}-c_{2}$ with the correspondent errors $\sigma_{1}$ and $\sigma_{2}$, respectively (see Figure 7).

Based on the WISE source location in the $c_{1}-c_{2}$ diagram we can distinguish five types of objects. Each source, given its IR colors, corresponds to a single point in each 2D color-color projection of the WGS. However, including the errors on both axes, it is represented by a cross with four extremal points, calculated considering the $\pm 1 \sigma$ error on each color. Then, we can define five different types of sources, according to the schematic view shown in Figure 7:

1. type 4: sources with all the extremal points within the WGS projection;

2. type 3: sources for which only three extremal points lie within the region of the WGS;

3. type 2: sources with only two extremal points consistent with the WGS;

4. type 1: sources with only a single extremal point associated with the WGS;

5. type 0: sources without extremal points of the error cross on the WGS.

We can assign to each type of source a discrete strip parameterd ranging between 0 and 1, according to the scheme illustrated in Figure 7. For example, in the case of the $c_{1}-c_{2}$ projection of the WGS, we assign to type 4 sources a value $d_{12}=1$, while source of type 0 corresponds to $d_{12}=0$. For the same 2D projection, the intermediate values have been assigned as follows: type 3 have $d_{12}=0.75$, type 2 have $d_{12}=0.5$, and type $1 d_{12}=0.25$.

On the same $c_{1}-c_{2}$ diagram, we also assign a weight strip parameter $w_{12}$ to each value of the $d_{12}$ parameter defined as $w_{12}=\left(\sigma_{1} \sigma_{2}\right)^{-1 / 2}$, proportional to the area of the ellipse described by the error bars of each point (see inset of Figure 7 for 

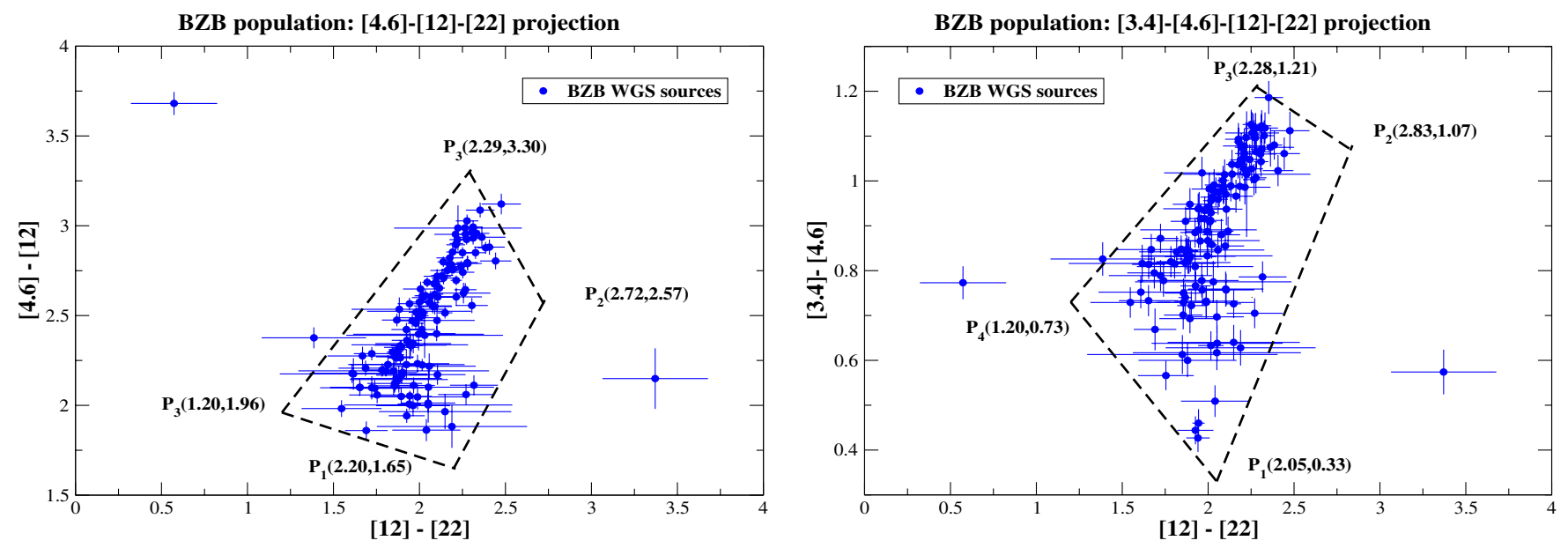

Figure 5. Same as Figure 3 for the case of the BZB population on the WGS in the two remaining different color-color projections.

(A color version of this figure is available in the online journal.)
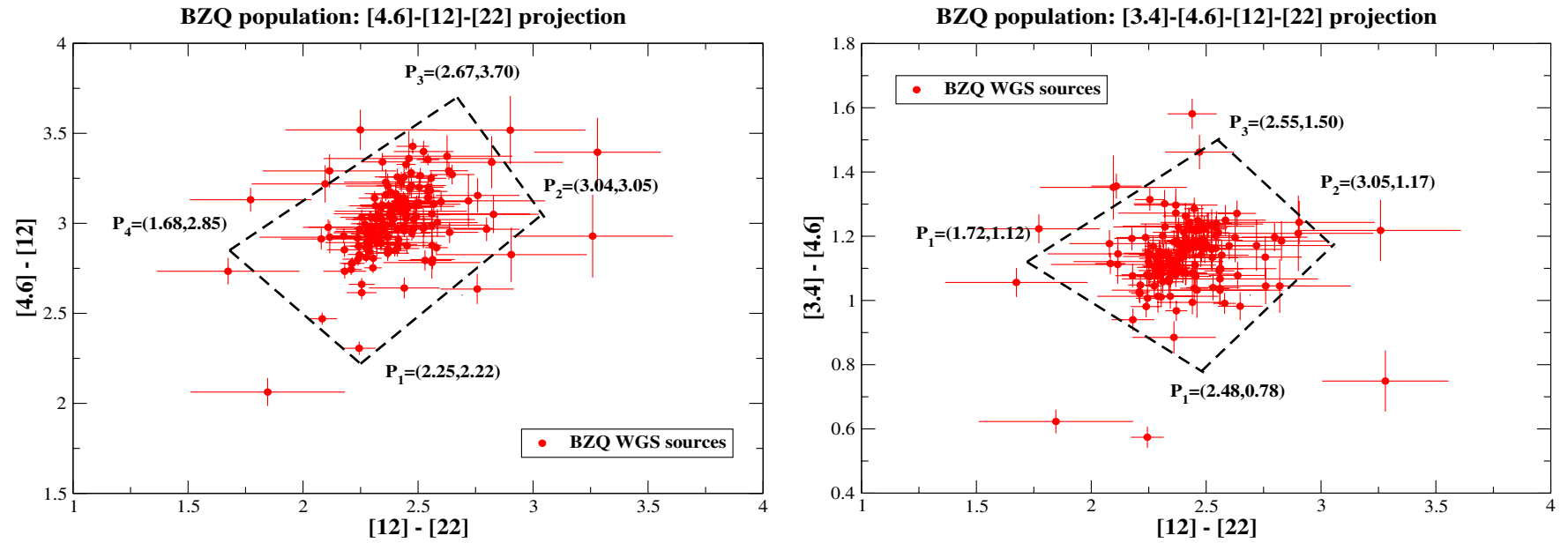

Figure 6. Same as Figure 4 for the case of the BZQ population on the WGS in the two remaining different color-color projections.

(A color version of this figure is available in the online journal.)

more details). Then, we define the continuous strip parameter $s_{12}$ as

$$
s_{12}=d_{12} w_{12}
$$

We note that the parameter $w_{12}$ has been chosen to take into account the different errors on both axes when comparing two sources that might belong to the same type. It also allows us to make the $s_{12}$ continuous rather than discrete as $d_{12}$.

We repeated the entire procedure described above for each $2 \mathrm{D}$ projection of the WGS: $c_{1}-c_{2}, c_{2}-c_{3}$, and $c_{1}-c_{3}$, generating the values of the continuous strip parameters $s_{12}, s_{23}, s_{13}$, respectively. Then, all these values of the strip parameters for three different 2D projections have been combined together to define a unique total strip parameter $s$. The total strip parameter is the geometric average of the $s$ values of each 2D projection:

$$
s=\left(s_{12} s_{23} s_{13}\right)^{1 / 3} .
$$

We emphasize that sources that lie outside of the WGS in at least one of its $2 \mathrm{D}$ projection have one of the correspondent $s_{12}, s_{23}, s_{13}$ parameter equal to zero and consequently the total $s$ value is null as well. This occurs because the discrete $d$ parameter is zero for sources outside the WGS (see Figure 7).

We divided all the $s$ parameters for the maximum $s$ values of the BZBs and BZQs that lie on the WGS to re-normalize $s$ in the range 0 and 1 . This re-normalization can be applied to the $s$ values of all the WISE sources, because those outside the WGS will have $s$ null by definition.

The $s$ parameter represents an estimate of the distance, in the IR colors' parameter space and weighted with the errors on each axes, between the WGS and a generic WISE source, that could potentially belong to it; this $s$ parameter is different from zero only in the case in which all color error bars of a WISE source are consistent with the WGS. Therefore, these $s$ values can be used to rank each IR WISE source according to their association with the WGS.

Finally, we note that to test if a generic WISE source has IR colors consistent with the BZBs or with the BZQs subregion of the WGS, the total strip parameters are referred to as $s_{b}$ and $s_{q}$, respectively. We introduced the above divisions for the $s$ parameters because in future works this allows us to verify if a generic WISE source is more consistent to be a BZB or a BZQ, enabling a classification for new IR sources that could lie on the WGS being $\gamma$-ray blazar candidates.

\subsection{The s Parameter Distributions}

We considered a sample composed of all the WISE sources lying in two circular regions of $1^{\circ}$ radius, centered at high and low Galactic latitude $b$, with the center coordinates of 


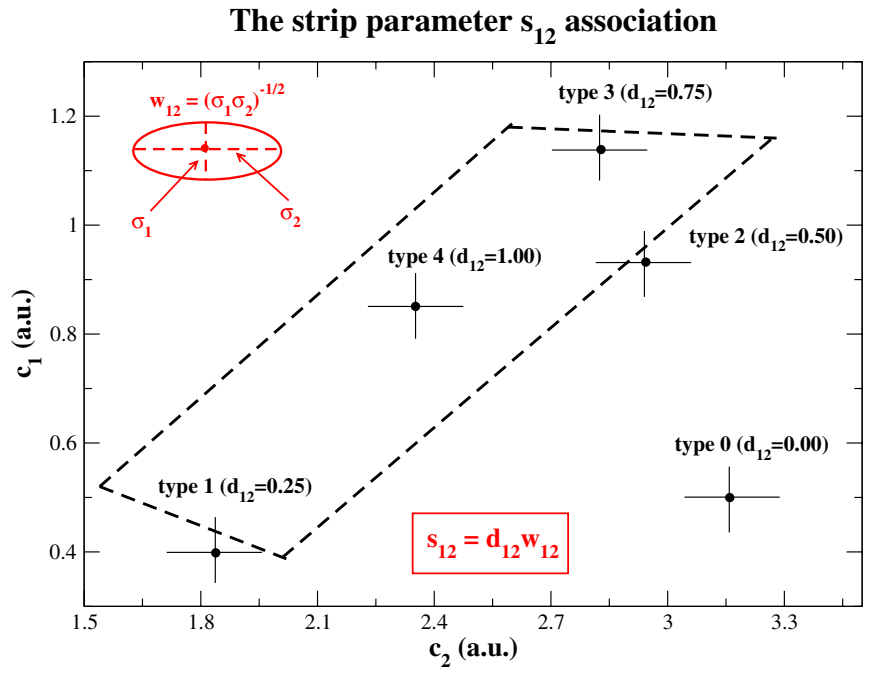

Figure 7. Schematic view of the strip parameterization in the example of the $c_{1}-c_{2} 2 \mathrm{D}$ projection in arbitrary units (a.u.). We report the method described in Section 2.4 to assign to each point of the $2 \mathrm{D}$ projection of the WGS a value of the discrete strip parameter $d_{12}$ and the associated value of the weight strip parameter $w_{12}$. The combination of these two values provides the continuous strip parameter $s_{12}$ for each given source (see Equation (1)).

(A color version of this figure is available in the online journal.)

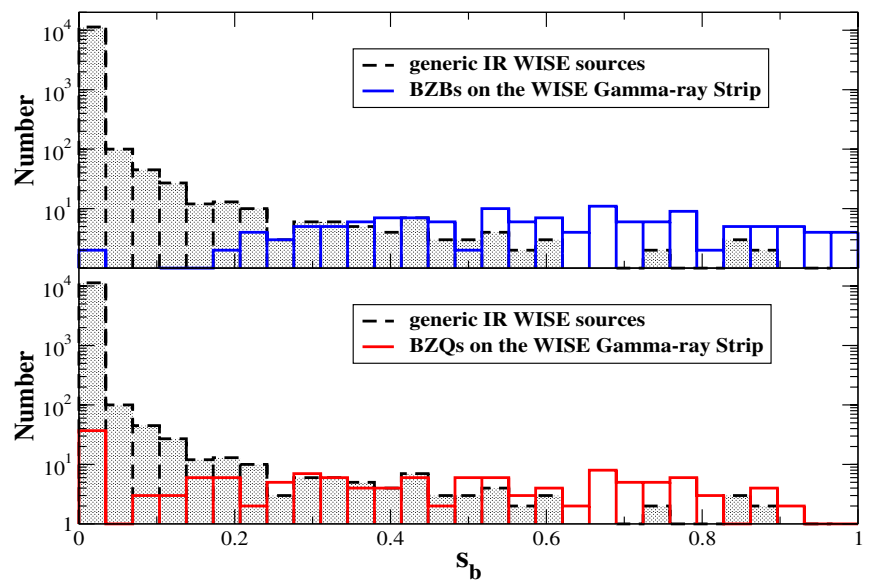

Figure 8. Distribution of the strip parameter $s_{b}$ for the BZBs (blue) and the BZQs (red) that lie on the WGS in comparison with the generic IR WISE sources (black).

(A color version of this figure is available in the online journal.)

$(l, b)=\left(255^{\circ},-55^{\circ}\right)$ and $(l, b)=\left(338^{\circ},-1^{\circ}\right)$, respectively. These sources do not have upper limits on their WISE magnitude values and are detected with a signal-to-noise ratio $>7$ in at least one band as for the blazars in the 2FB sample.

We calculated the $s$ parameters for all the 11,599 generic IR WISE sources. This analysis provides an estimate of the probability to find a generic WISE source in the sky with a particular value of $s_{b}$ and/or $s_{q}$. We perform this analysis considering the distinction between the two blazar classes (i.e., BZBs and BZQs). The distributions of the $s_{b}$ and $s_{q}$ parameters for the BZBs and the BZQs that lie on the WGS in comparison with the generic IR WISE sources are shown in Figures 8 and 9, respectively.

From the distributions of the $s_{b}$ and $s_{q}$ parameters for the generic IR WISE sources, we note that $99.9 \%$ of them have $s_{b}<0.24$ and $s_{q}<0.38$. Then, for the BZBs in the 2FB sample

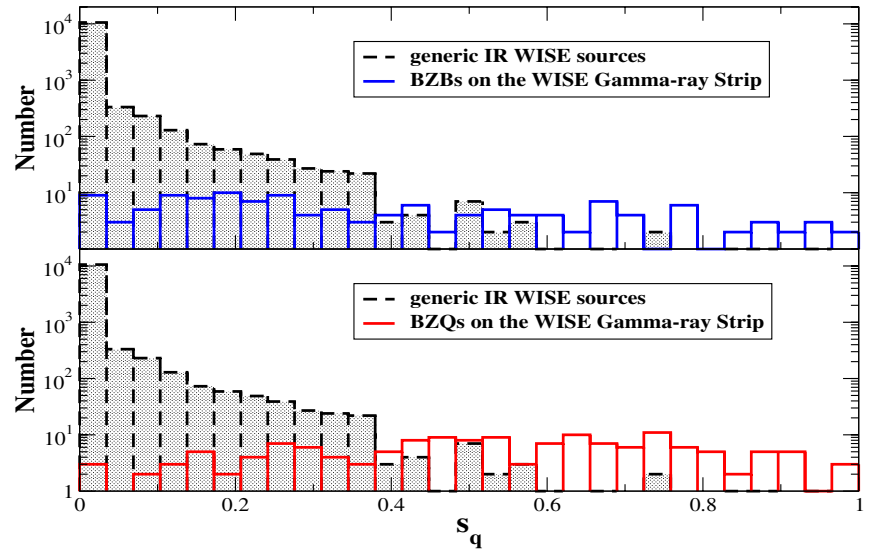

Figure 9. Same as Figure 8 for the distribution of the $s_{q}$ parameter. (A color version of this figure is available in the online journal.)

only 6 sources out of 135 have $s_{b}<0.24$, and in the case of the BZQs only 33 sources out of 149 show $s_{q}$ values lower than 0.38 . We also note that $99.0 \%$ of the generic IR WISE sources have $s_{b}<0.10$ and only 2 BZBs are below this value, while $97.2 \%$ of the generic IR WISE sources together with only 5 BZQs out of 149 have $s_{q}<0.14$. Finally, on the basis of the above $s$ distributions we define the outliers of the WGS, WISE sources that have values of $s_{b}<0.10$ or $s_{q}<0.14$.

We recognize that the above choice of the $s_{b}$ and $s_{q}$ thresholds is extremely conservative. This choice has been made on the basis of the actual sky coverage of the WISE preliminary data release. At the present status of our analysis we are not able to investigate the IR emission of all the blazars that are listed in the 2FGL, and the 2FB sample used to build the WGS parameterization is small with respect to how it will be available when the WISE full archive will be released. Consequently, regarding the choice of the threshold values for the $s$ parameters, we preferred the efficiency to the completeness of our method selecting the $s$ limiting values from their distributions in low galactic latitude regions, even if this choice could increase the possible contamination of the WGS. A deeper investigation of this problem will be considered in future as an a posteriori analysis of WGS parameterization. In particular, when the WISE full release will be available, we will improve our method taking into account the IR source density at different galactic latitudes and the varying depth of the exposure for the WISE observations.

\section{THE AGU COUNTERPARTS ON THE WGS}

We considered the sample composed of all the AGUs already classified in the analysis of the 2FGL and the 2LAC (Nolan et al. 2012; Ackermann et al. 2011). According to the 2FGL, the AGUs could be all blazar candidates without a good optical spectrum or without an optical spectrum at all (Nolan et al. 2012; Ackermann et al. 2011).

We selected the AGUs that lie in the portion of the sky surveyed by WISE during the first year, corresponding to 148 Fermi sources. Then, we excluded from our analysis all the AGUs with a Fermi analysis flag, according to the 2FGL and the 2LAC.

The association between each AGU counterpart and the WISE sources has been evaluated on the basis of the same criterion chosen for the blazars on the WISE Blazar Strip (see Section 2.2 and Paper I for more details), considering the position of the 


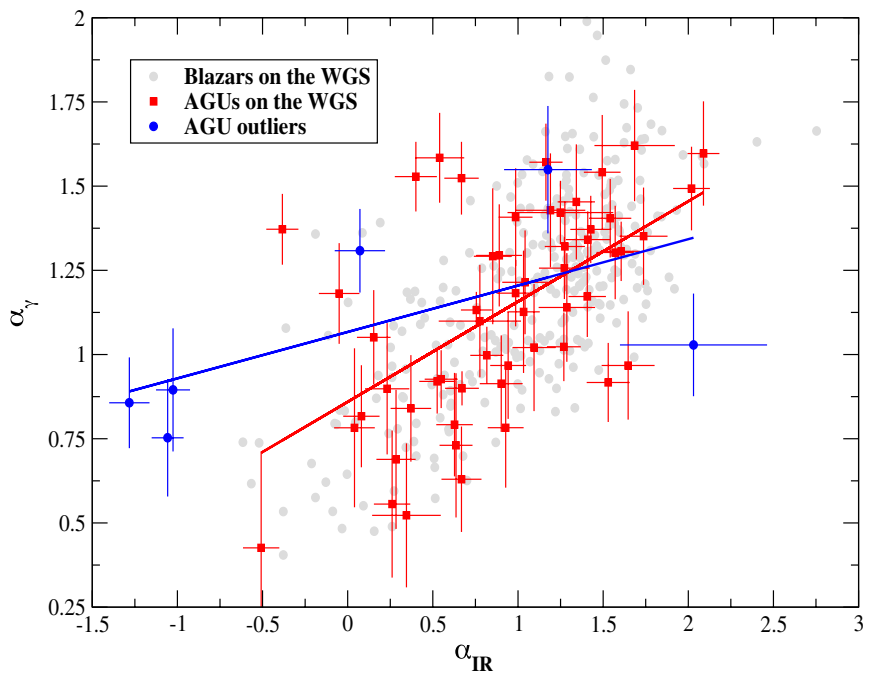

Figure 10. Correlation between $\alpha_{\mathrm{IR}}$ and $\alpha_{\gamma}$ for the WISE counterparts of the AGUs. The background gray circles represent the correlation found for the WGS blazars (see Paper II), while the red filled squares are the AGUs that have been found consistent with the WGS accordingly with our parameterization. The remaining six outliers (blue filled circles) show a weaker and different correlation than the other samples.

(A color version of this figure is available in the online journal.)

radio counterpart for each $\mathrm{AGU}$ as reported in the $2 \mathrm{FGL}$ and/or in the 2LAC. There are 60 AGUs out of 148 for which there is a unique association with a WISE source (see Section 3) within the usual region of 2". 4 radius and with a chance probability of 0.008 , estimated adopting the method described in Maselli et al. (2010; see also Maselli et al. 2011; Paper I) and without upper limits on the WISE magnitudes within the WISE preliminary data release.

Subsequently, we used the IR colors of the AGU counterparts, as associated in the 2LAC, to verify if the WISE counterparts of the $\gamma$-ray sources in the 60 AGU sample lie on the WGS, evaluating their $s$ values following the procedure described in Section 2.

We found that six outliers do not belong to the WGS out of 60 AGUs, according to the inclusion based on the threshold values of $s_{b}<0.10$ or $s_{q}<0.14$. With this analysis on the $60 \mathrm{AGU}$ sample, we have been able to check if the association provided by the 2FGL corresponds to a blazar lying on the WGS.

Finally, we estimated the IR spectral index $\alpha_{\mathrm{IR}}$ as parameterizing the [3.4]-[4.6] $\mu \mathrm{m}$ color according to Equation (1) of Paper II, and we evaluated the correlation between $\alpha_{\text {IR }}$ and the spectral index of the associated 2FGL source $\alpha_{\gamma}$. We found a linear correlation between $\alpha_{\mathrm{IR}}$ and $\alpha_{\gamma}$ for the $54 \mathrm{AGU}$ that lie on the WGS, with a correlation coefficient $\rho=0.56$ and a chance probability of $8.96 \times 10^{-6}$ and a slope $m=0.30 \pm 0.06$, that is consistent with that of the WGS blazars $(\rho=0.68, m=0.36$ \pm 0.02 , see Paper II) within $1 \sigma$ (see Figure 10). On the other hand, the six outliers have a weaker linear correlation between the two spectral indices then the previous sample with $\rho=0.40$ (chance probability of 0.08 ) and $m=0.12 \pm 0.07$, different from that of the blazars on the WGS (see Figure 10). Finally, in Tables 1 and 2 we report the colors, the IR spectral indices and the $s$ parameters together with the 2FGL name and the WISE and the counterpart names of each AGU analyzed. The class of each AGU as derived from the 2LAC analysis is also indicated (Ackermann et al. 2011).

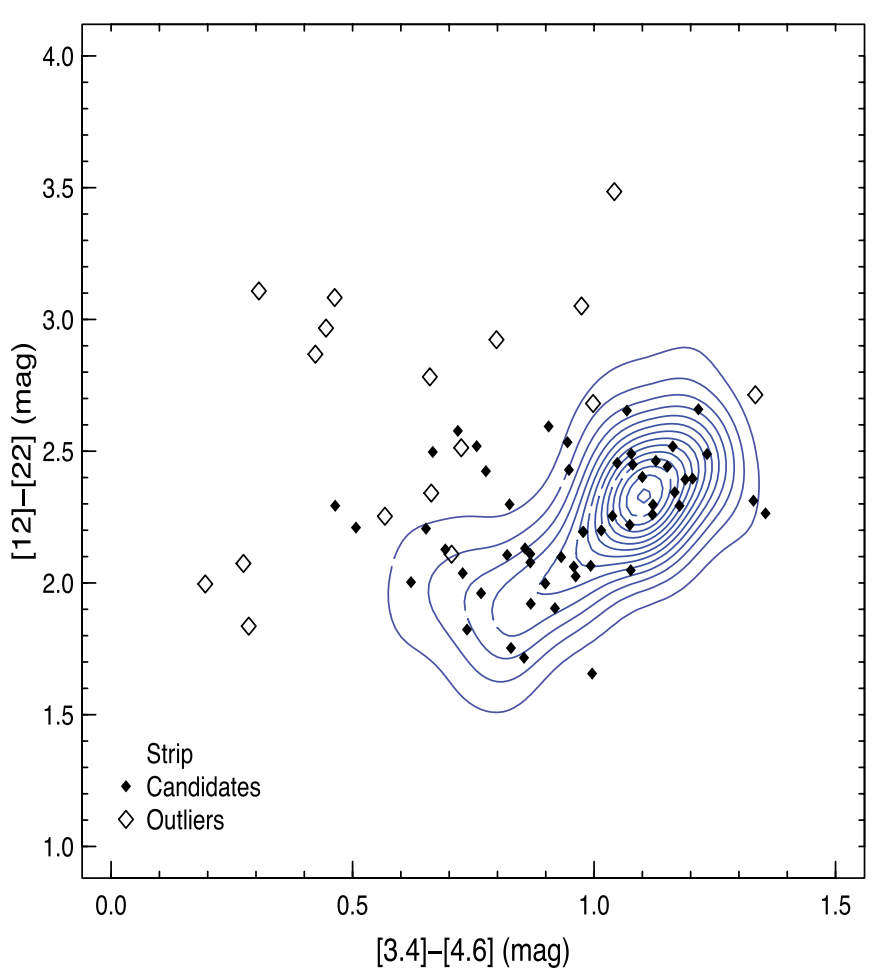

Figure 11. Isodensity contours drawn from the KDE technique for the blazars of the WGS (gray circles) are shown for the case of the [3.4]-[4.6]-[12]-[22] $\mu \mathrm{m}$ $2 \mathrm{D}$ projection. The AGU identified as blazar candidates (filled black squares) are also shown in comparison with the outliers (black open square) to show the consistency between the WGS parameterization and the KDE analysis.

(A color version of this figure is available in the online journal.)

\section{AN INDEPENDENT NON-PARAMETRIC ANALYSIS: THE KERNEL DENSITY ESTIMATION}

To test our analysis, we also performed a statistical investigation based on an independent non-parametric method as the KDE technique as already proposed in Paper I (see also D'Abrusco et al. 2009; Laurino \& D'Abrusco 2011 and reference therein). The KDE method provides an effective way of estimating the probability function of a multivariate distribution and does not require any assumption about the shape of the "parent" distributions. In Figure 11, the isodensity contours drawn from the KDE density probabilities and associated with different levels of density are plotted for the blazars of the WGS in its [3.4]-[4.6]-[12]-[22] $\mu \mathrm{m} 2 \mathrm{D}$ projection.

Consequently, for a generic source in the WISE archive we can provide an estimate of the probability $\pi_{\mathrm{kde}}$ that a blazar of the WGS has the same IR colors, this is a surrogate of the probability that a WISE source is consistent with the WGS. In Figure 11, we also show the AGU counterparts with respect to the isodensity contours of the WGSto highlight the outliers. We also report in Tables 1 and 2, the value of $\pi_{\mathrm{kde}}$ for each AGU analyzed.

Finally, we note that there are some AGUs for which the $\mathrm{KDE}$ analysis suggests that the source is not consistent with the WGS, even if the parametric method indicates it as a possible candidate. The reason for this to happen is that, as previously mentioned, the KDE method does not take into account the errors on the IR colors. As a consequence, sources far from the WGS but with large errors could be associated with lowdensity values, as calculated by the KDE method, and discarded. 
Table 1

Parameters of the WISE Counterparts for the Outliers of the WGS

\begin{tabular}{|c|c|c|c|c|c|c|c|c|c|c|c|c|c|c|}
\hline 2FGL Name & WISE Name & Name & Class & $c_{1}$ & $\sigma_{1}$ & $c_{2}$ & $\sigma_{2}$ & $c_{3}$ & $\sigma_{3}$ & $\alpha_{\mathrm{IR}}$ & $\sigma_{\alpha_{\mathrm{IR}}}$ & $s_{b}$ & $s_{q}$ & $\pi_{\mathrm{kde}}$ \\
\hline-7223 & J05334 & 16 & 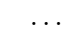 & 1.042 & .088 & 1.884 & 0.286 & 3.485 & 0.457 & 1.175 & 0.257 & 0.0 & 0.0 & $963 \mathrm{e}-$ \\
\hline GLJ0602.3+5315 & J060200 & GB6 J0 & HSP & 0.274 & 0.032 & 1.707 & 0.058 & 2.074 & 0.174 & -1.05 & 0.093 & 0.0 & 0.0 & $8 e-5$ \\
\hline 2FGLJ0605.0+0001 & J060458 & GB6 J06 & $\cdots$ & 0.285 & 0.034 & 1.218 & 0.089 & 1.836 & 0.431 & -1.02 & 0.099 & 0.0 & 0.0 & $2.09 \mathrm{e}-9$ \\
\hline 2FGLJ1753.8-5012 & J175338.55-501513.7 & PMN J1753-5015 & $\cdots$ & 0.663 & 0.050 & 2.647 & 0.057 & 2.341 & 0.122 & 0.071 & 0.147 & 0.0 & 0.0 & 0.003 \\
\hline 2FGLJ1936.9+8402 & J193739.76+835628.9 & $6 \mathrm{C} \mathrm{B} 194425+834912$ & $\cdots$ & 1.334 & 0.147 & 2.938 & 0.232 & 2.714 & 0.581 & 2.030 & 0.431 & 0.0 & 0.06 & 0.01 \\
\hline
\end{tabular}

Notes. Column 1: source name reported in the 2FGL (Nolan et al. 2012). Column 2: source name reported in the WISE preliminary data release. Column 3: source name as reported in the 2LAC (Ackermann et al. 2011). Column 4: source class as reported in the 2LAC: high-synchrotron-peaked blazar (HSP), intermediatedsynchrotron-peaked blazar (ISP), low-synchrotron-peaked blazar (LSP). Column 5: IR color $c_{1}=$ [3.4]-[4.6] $\mu \mathrm{m}$. Column 6: error $\sigma_{1}$ on $c_{1}$. Column 7: IR color $c_{2}=$ [4.6]-[12] $\mu \mathrm{m}$. Column 8: error $\sigma_{2}$ on $c_{1}$. Column 9: IR color $c_{3}=$ [12]-[22] $\mu \mathrm{m}$. Column 10: error $\sigma_{3}$ on $c_{3}$. Column 11: IR spectral index $\alpha$. Column 12: error on $\alpha_{\mathrm{IR}}$. Columns 13 and 14: $s_{b}$ and $s_{q}$ values. Column 15: probability derived from the KDE analysis $\pi_{\mathrm{kde}}$.

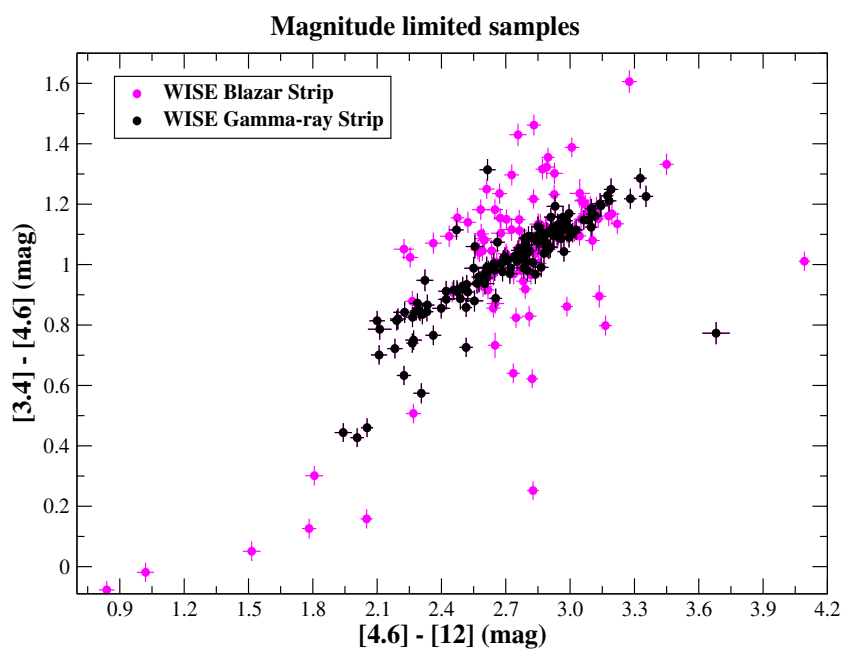

Figure 12. 2D projection of the WISE Blazar Strip (magenta) and the subregion of the WGS (black) in the IR color diagram [3.4]-[4.6]-[12] $\mu \mathrm{m}$ when only bright IR blazars are considered (i.e., those with WISE magnitudes in the ranges: $m_{1} \leqslant 13.5, m_{2} \leqslant 12, m_{3} \leqslant 11, m_{4} \leqslant 7.5$, respectively).

(A color version of this figure is available in the online journal.)

However, our parameterization of the WGS allows us to take into account the errors on the WISE colors and to consider also this type of sources. We emphasize that all the sources that the WGS parameterization indicates as outliers have also $\pi_{\mathrm{kde}}$ typically lower than $\sim 1 \%$ of being consistent with the WGS.

\section{AN ANALYSIS OF POSSIBLE SELECTION EFFECTS}

In the future, thanks to the developed WGS parameterization, we will also be able to investigate if there are selection effects that could affect our analysis as for example driving the WGS thickness. At the current stage of our study, we are able to estimate when a generic WISE sources in consistent with the WGS itself that is a necessary tool to compare different samples for future investigations; thanks to the parameterization developed we will also be able to verify if there are IR blazars that belong to the WGS but are not detected in $\gamma$-rays, and which could be the physical conditions if this occurs.

We remark that the link between the IR and the $\gamma$-ray properties of blazars is mainly due to the relation between the blazar spectral shape in the IR and in the $\gamma$-rays. To evaluate if selection effects due to flux limits in the selected sample could affect our WGS description, we performed the following tests. We restricted our analysis to the bright WISE blazars with IR

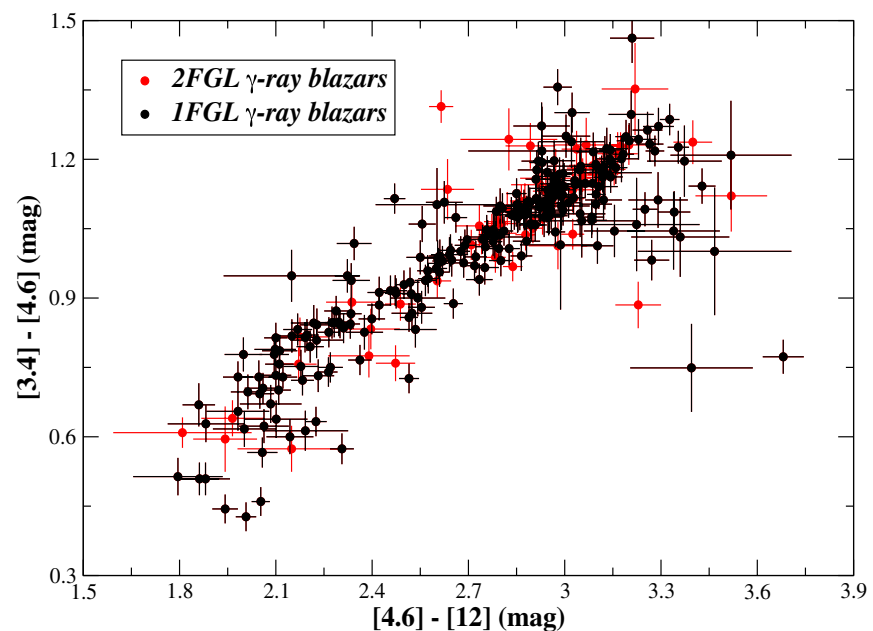

Figure 13. 2D projection of the WGS in the IR color diagram [3.4]-[4.6]-[12] $\mu \mathrm{m}$ when only bright $\gamma$-ray blazars listed in the 1FGL (black) are considered in comparison with those in the 2FGL (red). There are no clear differences on the thickness of the WGS in this 2D projection due to the different samples considered.

(A color version of this figure is available in the online journal.)

magnitudes in the following ranges: $m_{1} \leqslant 13.5, m_{2} \leqslant 12, m_{3} \leqslant$ $11, m_{4} \leqslant 7.5$, that belong to both the WISE Blazar Strip and to the WGS, and we found that a difference in their thickness is still evident (see Figure 12 for the standard 2D projection in [3.4]-[4.6]-[12] $\mu \mathrm{m}$ color diagram). This plot suggests that the origin of the WGS is not due to a selection of bright IR blazars.

We also compared the WGS as formed by the blazars present in the 2FGL and those detected in the first Fermi-LAT catalog (1FGL; Abdo et al. 2010), and again we did not find any clear difference between the WGS drawn with the bright or the faint $\gamma$-ray blazars. This again suggests that the relation between the WGS and the $\gamma$-ray detectability is related to the blazar spectral shape (see Figure 13 for the standard 2D projection in [3.4]-[4.6]-[12] $\mu \mathrm{m}$ color diagram). On the basis of the WGS parameterization presented in this paper, the above issues will be deeply addressed in future works, after the full release of the WISE all-sky survey.

\section{SUMMARY AND DISCUSSION}

On the basis of the recent results on the characterization of the IR colors of blazars provided by WISE (Paper I) and 
Table 2

Parameters of the WISE Counterparts for the Blazar Candidates

\begin{tabular}{|c|c|c|c|c|c|c|c|c|c|c|c|c|c|c|}
\hline 2FGL Name & WISE Name & Name & Class & $c_{1}$ & $\sigma_{1}$ & $c_{2}$ & $\sigma_{2}$ & $c_{3}$ & $\sigma_{3}$ & $\alpha_{\mathrm{IR}}$ & $\sigma_{\alpha_{\mathrm{IR}}}$ & $s_{b}$ & $s_{q}$ & $\pi_{\mathrm{kde}}$ \\
\hline 2FGLJ0156.4+3909 & J015631.40+391430.5 & MG4 J015630+3913 & 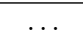 & 1.167 & 0.042 & 2.731 & 0.063 & 2.344 & 0.196 & 1.541 & 0.124 & 0.273 & 0.355 & 0.28 \\
\hline 2FGLJ0248.6+8440 & J024948.29+843556.9 & NVSS J024948+843556 & $\ldots$ & 0.958 & 0.036 & 2.654 & 0.039 & 2.062 & 0.098 & 0.927 & 0.105 & 0.548 & 0.475 & 0.41 \\
\hline 2FGLJ0253.4+3218 & J025333.64+321720.4 & MG3 J025334+3217 & $\cdots$ & 1.163 & 0.043 & 2.954 & 0.060 & 2.518 & 0.117 & 1.529 & 0.126 & 0.317 & 0.428 & 0.60 \\
\hline 2FGLJ0309.3-0743 & J030943.22-074427.5 & NVSS J030943-074427 & HSP & 0.728 & 0.036 & 2.128 & 0.071 & 2.037 & 0.248 & 0.260 & 0.106 & 0.327 & 0.109 & 0.18 \\
\hline 2FGLJ0332.5-1118 & J033223.25-111950.6 & NVSS J033223-111951 & HSP & 0.977 & 0.033 & 2.652 & 0.033 & 2.194 & 0.050 & 0.986 & 0.099 & 0.739 & 0.748 & 0.43 \\
\hline 2FGLJ0333.7+2918 & J033349.00+291631.6 & TXS 0330+291 & ISP & 0.820 & 0.036 & 2.096 & 0.048 & 2.106 & 0.134 & 0.525 & 0.105 & 0.460 & 0.0 & 0.09 \\
\hline 2FGLJ0424.3-5332 & $\mathrm{J} 042504.26-533158.3$ & PMN J0425-5331 & ISP & 0.993 & 0.032 & 2.614 & 0.031 & 2.065 & 0.050 & 1.032 & 0.095 & 0.762 & 0.733 & 0.41 \\
\hline 2FGLJ0438.8-4521 & $\mathrm{J} 043900.84-452222.2$ & PKS 0437-454 & LSP & 1.189 & 0.037 & 3.111 & 0.041 & 2.393 & 0.087 & 1.605 & 0.109 & 0.287 & 0.559 & 0.74 \\
\hline 2FGLJ0440.1-3211 & J043933.88-321009.8 & PKS 0437-322 & LSP & 0.757 & 0.069 & 2.701 & 0.148 & 2.519 & 0.422 & 0.344 & 0.201 & 0.114 & 0.078 & $4.12 \mathrm{e}-4$ \\
\hline 2FGLJ0440.4+1433 & J044021.13+143756.5 & TXS $0437+145$ & $\cdots$ & 1.234 & 0.048 & 3.020 & 0.068 & 2.489 & 0.163 & 1.737 & 0.140 & 0.0 & 0.354 & 0.43 \\
\hline 2FGLJ0456.5+2658 & J045617.36+270220.7 & MG2 J045613+2702 & $\cdots$ & 1.122 & 0.043 & 2.903 & 0.061 & 2.297 & 0.159 & 1.409 & 0.128 & 0.377 & 0.381 & 1.00 \\
\hline 2FGLJ0505.9+6116 & J050558.78+611335.6 & NVSS J050558+611336 & HSP & 0.766 & 0.041 & 1.948 & 0.085 & 1.961 & 0.347 & 0.371 & 0.119 & 0.211 & 0.0 & 0.11 \\
\hline 2FGLJ0508.1-1936 & J050818.99-193556.0 & PMN J0508-1936 & $\cdots$ & 1.048 & 0.070 & 2.901 & 0.151 & 2.455 & 0.474 & 1.190 & 0.205 & 0.128 & 0.150 & 0.59 \\
\hline 2FGLJ0525.5-6011 & J052542.42-601340.8 & SUMSS J052542-601341 & HSP & 0.718 & 0.044 & 2.268 & 0.125 & 2.577 & 0.369 & 0.231 & 0.129 & 0.163 & 0.093 & 0.01 \\
\hline 2FGLJ0526.8+6326 & J052606.71+631729.0 & GB6 J0526+6317 & $\cdots$ & 0.996 & 0.045 & 2.709 & 0.077 & 1.656 & 0.349 & 1.041 & 0.134 & 0.193 & 0.140 & 0.02 \\
\hline 2FGLJ0532.0-4826 & J053158.61-482736.1 & PMN J0531-4827 & LSP & 0.899 & 0.031 & 2.492 & 0.029 & 1.998 & 0.039 & 0.755 & 0.090 & 0.862 & 0.457 & 0.37 \\
\hline 2FGLJ0537.7-5716 & J053748.95-571830.0 & SUMSS J053748-571828 & ISP & 0.857 & 0.033 & 2.348 & 0.041 & 2.131 & 0.112 & 0.636 & 0.096 & 0.530 & 0.281 & 0.17 \\
\hline 2FGLJ0538.5-3909 & J053810.35-390842.5 & 1RXS 053810.0-390839 & HSP & 0.621 & 0.041 & 2.032 & 0.118 & 2.003 & 0.492 & -0.050 & 0.119 & 0.194 & 0.061 & 0.13 \\
\hline 2FGLJ0604.2-4817 & J060408.61-481724.9 & 1ES 0602-482 & HSP & 0.666 & 0.036 & 1.943 & 0.085 & 2.497 & 0.224 & 0.080 & 0.106 & 0.233 & 0.0 & 0.006 \\
\hline 2FGLJ0609.4-0248 & J060915.06-024754.6 & NVSS J060915-024754 & HSP & 0.855 & 0.036 & 2.218 & 0.064 & 1.716 & 0.321 & 0.627 & 0.107 & 0.284 & 0.130 & 0.21 \\
\hline 2FGLJ0621.9+3750 & J062157.63+375057.4 & GB6 J0621+3750 & $\cdots$ & 1.204 & 0.053 & 3.096 & 0.070 & 2.396 & 0.161 & 1.646 & 0.155 & 0.164 & 0.339 & 0.68 \\
\hline 2FGLJ0703.1-3912 & J070312.64-391418.7 & NVSS J070312-391418 & ISP & 0.962 & 0.036 & 2.602 & 0.04 & 2.025 & 0.105 & 0.942 & 0.105 & 0.531 & 0.436 & 0.40 \\
\hline 2FGLJ0706.5+7741 & J070651.32+774137.1 & NVSS J070651+774137 & ISP & 0.919 & 0.033 & 2.489 & 0.034 & 1.904 & 0.079 & 0.816 & 0.097 & 0.628 & 0.264 & 0.31 \\
\hline 2FGLJ0734.2-7706 & J073443.42-771114.1 & PKS 0736-770 & LSP & 1.128 & 0.041 & 3.165 & 0.052 & 2.463 & 0.134 & 1.427 & 0.120 & 0.334 & 0.435 & 0.59 \\
\hline 2FGLJ0823.0+4041 & J082257.55+404149.8 & B3 0819+408 & LSP & 1.177 & 0.037 & 2.963 & 0.041 & 2.293 & 0.072 & 1.570 & 0.109 & 0.512 & 0.596 & 0.70 \\
\hline 2FGLJ0856.0+7136 & J085654.85+714623.4 & GB6 J0856+7146 & LSP & 1.152 & 0.036 & 3.007 & 0.04 & 2.442 & 0.071 & 1.494 & 0.107 & 0.450 & 0.608 & 0.88 \\
\hline 2FGLJ1021.6+8021 & $\mathrm{J} 102202.09+802349.9$ & WN B1016.6+8038 & $\ldots$ & 0.945 & 0.046 & 2.741 & 0.083 & 2.534 & 0.212 & 0.889 & 0.134 & 0.278 & 0.263 & 0.06 \\
\hline 2FGLJ1029.9+7437 & $\mathrm{J} 103122.15+744157.0$ & S5 $1027+74$ & ISP & 0.507 & 0.032 & 2.269 & 0.035 & 2.210 & 0.063 & -0.383 & 0.094 & 0.387 & 0.0 & 0.01 \\
\hline 2FGLJ1304.3-4353 & $\mathrm{J} 130421.02-435310.1$ & 1RXS 130421.2-435308 & HSP & 0.869 & 0.034 & 2.268 & 0.032 & 1.921 & 0.053 & 0.671 & 0.099 & 0.727 & 0.305 & 0.30 \\
\hline 2FGLJ1351.3-2909 & J135146.86-291217.5 & PKS 1348-289 & $\cdots$ & 1.077 & 0.040 & 2.885 & 0.059 & 2.49 & 0.137 & 1.275 & 0.117 & 0.392 & 0.415 & 0.62 \\
\hline 2FGLJ1406.2-2510 & J140609.61-250808.6 & NVSS J140609-250808 & $\cdots$ & 0.868 & 0.040 & 2.461 & 0.067 & 2.110 & 0.228 & 0.668 & 0.117 & 0.333 & 0.209 & 0.24 \\
\hline 2FGLJ1407.5-4257 & J140739.73-430231.9 & CGRaBS J1407-4302 & LSP & 0.948 & 0.043 & 2.724 & 0.066 & 2.429 & 0.149 & 0.901 & 0.125 & 0.377 & 0.365 & 0.12 \\
\hline 2FGLJ1416.0+1323 & $\mathrm{J} 141558.82+132023.8$ & PKS B1413+135 & LSP & 1.355 & 0.031 & 2.897 & 0.030 & 2.264 & 0.038 & 2.089 & 0.093 & 0.0 & 0.766 & 0.03 \\
\hline 2FGLJ1416.3-2415 & J141612.18-241813.4 & NVSS J141612-241812 & HSP & 0.464 & 0.036 & 1.702 & 0.093 & 2.293 & 0.277 & -0.508 & 0.106 & 0.141 & 0.0 & 0.003 \\
\hline 2FGLJ1419.4+7730 & $\mathrm{J} 141900.31+773228.8$ & 1RXS 141901.8+773229 & HSP & 0.652 & 0.041 & 2.013 & 0.116 & 2.206 & 0.493 & 0.039 & 0.119 & 0.195 & 0.0 & 0.09 \\
\hline 2FGLJ1419.4-0835 & $\mathrm{J} 141922.56-083832.1$ & NVSS J141922-083830 & LSP & 1.121 & 0.037 & 2.885 & 0.045 & 2.26 & 0.091 & 1.406 & 0.109 & 0.528 & 0.534 & 0.92 \\
\hline 2FGLJ1421.1-1117 & $\mathrm{J} 142100.15-111820.1$ & PMN J1420-1118 & $\cdots$ & 1.080 & 0.056 & 2.714 & 0.122 & 2.449 & 0.318 & 1.286 & 0.165 & 0.208 & 0.207 & 0.39 \\
\hline 2FGLJ1518.2-2733 & $\mathrm{J} 151803.60-273131.0$ & TXS 1515-273 & $\cdots$ & 0.692 & 0.033 & 2.190 & 0.044 & 2.127 & 0.097 & 0.153 & 0.098 & 0.539 & 0.180 & 0.11 \\
\hline 2FGLJ1553.2-2424 & J155331.63-242205.7 & PKS 1550-242 & $\cdots$ & 1.100 & 0.037 & 3.058 & 0.045 & 2.402 & 0.090 & 1.342 & 0.109 & 0.506 & 0.536 & 0.85 \\
\hline 2FGLJ1558.3+8513 & $\mathrm{J} 160031.78+850949.2$ & WN B1609.6+8517 & LSP & 0.868 & 0.034 & 2.301 & 0.043 & 2.078 & 0.132 & 0.668 & 0.100 & 0.487 & 0.234 & 0.21 \\
\hline 2FGLJ1558.6-7039 & J155736.14-704027.1 & PKS 1552-705 & $\cdots$ & 0.906 & 0.082 & 3.042 & 0.151 & 2.594 & 0.354 & 0.776 & 0.241 & 0.0 & 0.142 & 0.01 \\
\hline 2FGLJ1725.1-7714 & J172350.85-771349.9 & PKS 1716-771 & $\cdots$ & 1.038 & 0.033 & 2.769 & 0.040 & 2.254 & 0.092 & 1.164 & 0.097 & 0.568 & 0.575 & 0.72 \\
\hline 2FGLJ1759.2-4819 & J175858.45-482112.6 & PMN J1758-4820 & $\cdots$ & 0.978 & 0.038 & 2.609 & 0.036 & 2.194 & 0.057 & 0.986 & 0.112 & 0.655 & 0.635 & 0.39 \\
\hline 2FGLJ1816.7-4942 & $\mathrm{J} 181656.00-494344.0$ & PMN J1816-4943 & $\cdots$ & 1.076 & 0.051 & 2.868 & 0.066 & 2.048 & 0.213 & 1.272 & 0.149 & 0.274 & 0.320 & 0.32 \\
\hline 2FGLJ1818.7+2138 & $\mathrm{J} 181905.22+213234.0$ & MG2 J181902+2132 & $\cdots$ & 0.932 & 0.038 & 2.464 & 0.055 & 2.098 & 0.187 & 0.851 & 0.111 & 0.386 & 0.261 & 0.31 \\
\hline 2FGLJ1825.1-5231 & $\mathrm{J} 182513.82-523057.7$ & PKS 1821-525 & $\cdots$ & 1.074 & 0.034 & 2.864 & 0.034 & 2.220 & 0.067 & 1.269 & 0.099 & 0.658 & 0.666 & 0.87 \\
\hline 2FGLJ1829.1+2725 & J182913.97+272902.9 & 87 GB $182712.0+272717$ & $\cdots$ & 0.776 & 0.042 & 2.796 & 0.059 & 2.424 & 0.157 & 0.400 & 0.123 & 0.0 & 0.147 & $8.65 e-4$ \\
\hline 2FGLJ1830.0+1325 & $\mathrm{J} 183000.76+132414.4$ & MG1 J183001+1323 & $\cdots$ & 1.015 & 0.043 & 2.631 & 0.047 & 2.199 & 0.122 & 1.094 & 0.126 & 0.451 & 0.457 & 0.46 \\
\hline 2FGLJ1858.1-2510 & $\mathrm{J} 185819.07-251050.5$ & PMN J1858-2511 & $\cdots$ & 1.068 & 0.106 & 3.183 & 0.100 & 2.654 & 0.179 & 1.248 & 0.310 & 0.123 & 0.231 & 0.14 \\
\hline
\end{tabular}


Table 2

(Continued)

\begin{tabular}{|c|c|c|c|c|c|c|c|c|c|c|c|c|c|c|}
\hline 2FGL Name & WISE Name & Name & Class & $c_{1}$ & $\sigma_{1}$ & $c_{2}$ & $\sigma_{2}$ & $c_{3}$ & $\sigma_{3}$ & $\alpha_{\mathrm{IR}}$ & $\sigma_{\alpha_{\mathrm{IR}}}$ & $s_{b}$ & $s_{q}$ & $\pi_{\mathrm{kde}}$ \\
\hline 2FGLJ1913.4+4440 & $\mathrm{J} 191401.88+443832.5$ & 1RXS 191401.9+443849 & HSP & 0.737 & 0.039 & 2.256 & 0.081 & 1.823 & 0.400 & 0.283 & 0.115 & 0.249 & 0.125 & 0.20 \\
\hline 2FGLJ1940.8-6213 & J194121.77-621120.8 & PKS 1936-623 & & 1.330 & 0.036 & 2.860 & 0.043 & 2.312 & 0.092 & 2.018 & 0.107 & 0.0 & 0.542 & 0.04 \\
\hline 2FGLJ1941.6+7218 & $\mathrm{J} 194127.01+722142.2$ & 87GB 194202.1+721428 & $\cdots$ & 1.216 & 0.080 & 3.383 & 0.124 & 2.659 & 0.271 & 1.684 & 0.236 & 0.0 & 0.194 & 0.13 \\
\hline 2FGLJ1959.6-2931 & J200016.96-293026.2 & PMN J2000-2931 & $\ldots$ & 0.825 & 0.049 & 2.398 & 0.094 & 2.298 & 0.304 & 0.539 & 0.144 & 0.241 & 0.147 & 0.04 \\
\hline 2FGLJ2103.6-6236 & $\mathrm{J} 210338.37-623225.5$ & PMN J2103-6232 & HSP & 0.828 & 0.033 & 2.312 & 0.037 & 1.753 & 0.132 & 0.548 & 0.096 & 0.516 & 0.217 & 0.26 \\
\hline
\end{tabular}

Notes. Column 1: source name reported in the 2FGL (Nolan et al. 2012). Column 2: source name reported in the WISE preliminary data release. Column 3: source name as reported in the 2LAC (Ackermann et al. 2011). Column 4: source class as reported in the 2LAC: high-synchrotron-peaked blazar (HSP), intermediated-synchrotron-peaked blazar (ISP), low-synchrotron-peaked blazar (LSP). COlumn 5: IR color $c_{1}=$ [3.4]-[4.6] $\mu \mathrm{m}$. Column 6: error $\sigma_{1}$ on $c_{1}$. Column 7: IR color $c_{2}=$ [4.6]-[12] $\mu \mathrm{m}$. CColumn 8: error $\sigma_{2}$ on $c_{1}$. COlumn 9: IR color $c_{3}=$ [12]-[22] $\mu \mathrm{m}$. Column 10: error $\sigma_{3}$ on $c_{3}$. Column 11: IR spectral index $\alpha_{\mathrm{IR}}$. Column 12: error on $\alpha_{\mathrm{IR}}$. Columns 13 and 14: $s_{b}$ and $s_{q}$ values. Column 15: probability derived from the KDE analysis $\pi_{\mathrm{kde}}$. 
on the comparison with their $\gamma$-ray emission (Paper II), we developed a method based the WISE Blazar Strip to identify blazar counterparts of $\gamma$-ray sources.

We developed a method to parameterize the WGS in the $3 \mathrm{D}$ color diagrams based on its $2 \mathrm{D}$ projections. This method is characterized by the use of a continuous parameter $s$, in the range $0-1$, that takes into account the errors of all the IR colors and provides clues on the position of a generic WISE source relative to the WGS in the 3D color parameter space. High values of the $s$ parameters are associated with sources that lie inside the WGS (as the $\gamma$-ray blazar population of the 2FB sample). The WGS has been parameterized in two subregions, the first containing the BZBs while the other with the BZQs, although in the present work we are only interested in searching for blazar counterparts that lie on the WGS.

We applied our parameterization to the sample of the AGUs selected from the 2FGL and the 2LAC. We found that there are 148 AGUs that can be analyzed within the footprint of the WISE preliminary source catalog. However, according to our association procedure (see Section 2.2) only 60 AGUs have a unique WISE counterpart without any upper limit on the WISE magnitude values.

Then, we calculated the distributions of their $s$ parameter and found that 54 out of 60 AGUs analyzed are consistent with the WGS, corresponding to the $90 \%$ of the $\gamma$-ray counterparts analyzed while the remaining 6 AGU counterparts are outliers of the WGS. In particular, for the 54 AGUs that are consistent with the WGS, we also found that the correlation between the $\alpha_{\mathrm{IR}}$ and $\alpha_{\gamma}$ is in agreement with that found for the blazars that constitute the WGS itself while the same correlation for the six outliers is inconsistent with it.

We also applied the KDE non-parametric test to obtain the probability that an AGU counterpart belongs to the WGS and we found consistent results with our parameterization (see Section 4 for more details).

In addition, an extensive investigation of all the unidentified $\gamma$-ray sources in the 2FGL that fall in the area of the sky where the WISE preliminary data have been already released will be provided in a forthcoming paper (Massaro et al. 2012). Searching for blazar candidates within the unidentified $\gamma$-ray source sample could potentially leading to the discovery new class of $\gamma$-ray emitting sources.

Further improvements of the WGS parameterization will also be possible in the future, when the whole WISE catalog will be available and this parametric method would be calibrated at different $b$ values not only to look for counterparts of $\gamma$-ray sources but also to search for new blazar candidates all over the sky (Massaro et al. 2012, in press).

We are grateful to the anonymous referee for several constructive comments that have been helpful in improving our presentation. F. Massaro thanks A. Cavaliere, S. Digel, M. Elvis, D. Harris, J. Knodlseder, and D. Thompson for their fruitful discussions, and P. Giommi for his help with the ROMA-BZCAT analysis. F. Massaro also thanks D. Weedman for his helpful suggestions on the starburst galaxies. The work at SAO is supported in part by the NASA grants NNX10AD50G and NNX10AD68G. R. D'Abrusco gratefully acknowledges the financial support of the U.S. Virtual Astronomical Observatory, which is sponsored by the National Science Foundation and the National Aeronautics and Space Administration. F. Massaro acknowledges the Fondazione Angelo Della Riccia for the grant awarded him to support his research at SAO during 2011 and the Foundation BLANCEFLOR BoncompagniLudovisi, n'ee Bildt, for the grant awarded him in 2010 to support his research. TOPCAT (www.star.bris.ac.uk/ mbt/topcat/; Taylor 2005) was used extensively in this work for the preparation and manipulation of the tabular data. Part of this work is based on archival data, software or online services provided by the ASI Science Data Center. This publication makes use of data products from the Wide-field Infrared Survey Explorer, which is a joint project of the University of California, Los Angeles, and the Jet Propulsion Laboratory/California Institute of Technology, funded by the National Aeronautics and Space Administration.

\section{REFERENCES}

Abdo, A. A., Ackermann, M., Ajello, M., et al. 2010, ApJS, 188, 405 Ackermann, M., Ajello, M., Allafort, A., et al. 2011, ApJ, 743, 171

Blandford, R. D., \& Rees, M. J. 1978, in Proc. Pittsburgh Conf. on BL Lac Objects (Pittsburgh, PA: Univ. of Pittsburgh), 328

Cutri, R. M., et al. 2011, Wise Report, 1

D’ Abrusco, R., Longo, G., \& Walton, N. A. 2009, MNRAS, 396, 223

D'Abrusco, R., Massaro, F., Ajello, M., et al. 2012, ApJ, 748, 68

Laurino, O., D'Abrusco, R., Longo, G., \& Riccio, G. 2011, MNRAS, 418, 2165

Maselli, A., Cusumano, G., Massaro, E., et al. 2011, A\&A, 531, 153

Maselli, A., Massaro, E., Nesci, R., et al. 2010, A\&A, 512A, 74

Massaro, E., Giommi, P., Leto, C., et al. 2009, A\&A, 495, 691

Massaro, E., Giommi, P., Leto, C., et al. 2011, in Multifrequency Catalogue of Blazars (3rd Edition), ed. E. Massaro, P. Giommi, C. Leto et al. (Rome: ARACNE Editrice)

Massaro, E., Giommi, P., Leto, C., et al. 2010, arXiv:1006.0922

Massaro, F., D’Abrusco, R., Ajello, M., Grindlay, J. E., \& Smith, H. A. 2011, ApJ, 740, L48

Massaro, F., D'Abrusco, R., Tosti, G., et al. 2012, ApJ, in press

Nolan, P. L., Abdo, A. A., Ackermann, M., et al. 2012, ApJS, 199, 31

Plotkin, R. M., Anderson, S. F., Brandt, M. N., et al. 2011, ApJ, 745, L27

Stickel, M., Padovani, P., Urry, C. M., Fried, J. W., \& Kuehr, H. 1991, ApJ, 374, 431

Stocke, J. T., Morris, S. L., Gioia, I. M., et al. 1991, ApJS, 76, 813

Taylor, M. B. 2005, in ASP Conf. Ser. 347, Astronomical Data Analysis Software and Systems XIV, ed. P. Shopbell, M. Britton, \& R. Ebert (San Francisco, CA: ASP), 29

Wright, E. L., Eisenhardt, P. R. M., Mainzer, A. K., et al. 2010, AJ, 140, 1868 